\title{
Lymphangiopathy in neurofibromatosis I manifesting with chylothorax, pericardial effusion, and leg edema
}

This article was published in the following Dove Press journal:

International Journal of General Medicine

7 September 2013

Number of times this article has been viewed

\author{
Josef Finsterer ${ }^{1}$ \\ Claudia Stollberger ${ }^{2}$ \\ Elisabeth Stubenberger ${ }^{3}$ \\ Sasan Tschakoschian ${ }^{4}$ \\ 'Krankenanstalt Rudolfstiftung, \\ Vienna, Austria; ${ }^{2}$ Medical Department, \\ Krankenanstalt Rudolfstiftung, \\ Vienna, Austria; ${ }^{3}$ Thoracic Surgery \\ Department, Vienna, Austria; ${ }^{4}$ Interne \\ Lungenabt, Vienna, Austria
}

Background: This case report documents the affliction of the lymph vessels as a phenotypic feature of neurofibromatosis-1 (NF-1).

Methodology: Routine transthoracic echocardiography, computed tomography scan of the thorax, magnetic resonance angiography of the renal arteries, and conventional digital subtraction angiography were applied. Comprehensive NF-1 mutation analysis was carried out by fluorescence in situ hybridization analysis, long-range reverse transcriptase polymerase chain reaction, and multiple-ligation probe assay. All other investigations were performed using routine, well-established techniques.

Results: The subject is a 34-year-old, half-Chinese male; NF-1 was suspected at age 15 years for the first time. His medical history included preterm birth, mild facial dysmorphism, "café au lait" spots, subcutaneous and paravertebral fibromas, multifocal tachycardia, atrial fibrillation, and heart failure in early infancy. Noncalcified bone fibromas in the femur and tibia were detected at age 8 years. Surgical right leg lengthening was carried out at age 11 years. Bilateral renal artery stenosis, stenosis and aneurysm of the superior mesenteric artery, and an infrarenal aortic stenosis were detected at age 15 years. Leg edema and ectasia of the basilar artery were diagnosed at age 18 years. After an episode with an erysipela at age 34 years, he developed pericardial and pleural effusion during a 4-month period. Stenosis of the left subclavian vein at the level of thoracic duct insertion was detected. After repeated pleural punctures, pleural effusion was interpreted as chylothorax. Reduction of lymph fluid production by diet and injection of talcum into the pleural cavity had a long-term beneficial effect on the chylothorax. Leg edema and chylothorax were attributed to affliction of the lymph vessels by the NF-1.

Discussion: Lymphangiopathy resulting in impaired lymph fluid flow and sequestration of lymph fluid into the pleural sinus and the legs may be a rare phenotypic feature of NF-1.

Keywords: angiodysplasia, lymph edema, lymph fluid, Recklinghausen's disease, genetics, mutation

\section{Background}

Neurofibromatosis type-I (NF-I) is an autosomal dominant RASopathy affecting one in 3000 individuals. ${ }^{1}$ Other RASopathies include Noonan syndrome, Costello syndrome, LEOPARD syndrome, cardiofaciocutaneous syndrome, or Leopold syndrome. Vascular anomalies, predominantly affecting the arteries, are well-recognized manifestations of NF- $\mathrm{I}^{1}$ and manifest as aneurysms, arterial stenoses, arteriovenous malformations, arteries compressed or invaded by neural tumors, or heart valve abnormalities. ${ }^{1}$ Abnormalities of the lymph vessels, in particular the thoracic duct, as in the following case, have been only rarely reported. ${ }^{2}$
Correspondence: Josef Finsterer Krankenanstalt Rudolfstiftung, Postfach 20, II80 Vienna, Austria Tel +43 | 7 I I65 92085

Fax +43 I 478I7II

Email fifigs I@yahoo.de 


\section{Case report}

The patient is a 34-year-old, non-smoking, HIV-negative, half-Chinese male, weighing $103 \mathrm{~kg}$, height of $186 \mathrm{~cm}$, with a medical history including preterm birth, congenital "café au lait" macules (CALM) in the right half of the face and the right trunk, multifocal tachycardia, atrial fibrillation, and heart failure between birth and 8 months. At age 8 years, noncalcified, bilateral bone fibromas were detected in the femur and tibia, and orchidopexy and herniotomy were carried out. Surgical right leg lengthening was performed at age 11 years. At the age of 15 years arterial hypertension was noted, and diagnostic work-up revealed bilateral renal artery stenosis, and both stenosis and aneurysm of the superior mesenteric artery. An infrarenal aortic stenosis was successfully dilated. Marked varicosities of the lower legs developed. Based upon these abnormalities, NF-1 was suspected for the first time. Since the age of 18 years, lymphatic edema of both lower legs developed, and ectasia of the basilar artery was found on cerebral computed tomography (CT). He experienced his first tonic-clonic seizure at the age of 28 years, followed by recurrent absences at age 32 years. He received levetiracetam for seizures, but discontinued it 2 weeks later, opting to treat the epilepsy with acupuncture instead. The family history was negative for NF-1.

In June 2011, at the age of 34 years, he experienced a minimal trauma to the distal lower left leg while in Turkey, and consecutively developed fever and erysipelas. Echocardiography revealed dilation, systolic dysfunction, increased pulmonary pressure, and pericardial effusion of $10 \mathrm{~mm}$. During the flight back home he experienced his second generalized tonic-clonic seizure in his life, believed to have been triggered by sleep deprivation. On admission, $\mathrm{X}$-ray of the lungs showed bronchitis and suspected interstitial fibrosis. Hypothyroidism with struma diffusa and struma nodosa were additionally detected. In addition to the left renal artery stenosis, magnetic resonance angiography of the renal arteries revealed a splenorenal shunt, hepatosplenomegaly, a liver cyst (segment $\mathrm{V}$ ), and right-sided renal cysts. Magnetic resonance angiography of the abdominal aorta in July 2011 showed moderate stenosis of the left and high-grade stenosis of the doubled right renal artery, elongated external iliac and common femoral artery on the right side, and strong lumbar arteries with collaterals to the inferior epigastric artery. Additionally, prevertebral neurofibromas were detected along the thoracic spine.

In August 2011 he was admitted for thoracic pain. Chest X-ray showed an enlarged heart and left pleural effusion. Transthoracic echocardiography revealed slight left ventricular myocardial thickening, bilaterally enlarged atria, mitral valve insufficiency, and slight pericardial effusion. Puncture of the pleura was indicative of inflammation and graded as PAPIII. Clinical neurologic investigation in August 2011 revealed hypertelorism, slight distal wasting on the upper limbs, exaggerated tendon reflexes on the left upper limb, reduced patella tendon reflexes, lymphedema of the left lower limb, and some subcutaneous fibromas on the upper limbs. Cerebral magnetic resonance imaging showed elongation of the vertebral arteries exclusively.

DNA analysis of the NF-1 gene was carried out in August 2011. Comprehensive NF-1 mutation analysis of leukocytic DNA initially aimed at identifying microsatellite polymorphisms. Since no heterozygous signal was detected, fluorescence in situ hybridization analysis was performed. The total coding region of the NF-1 gene was analyzed by long-range reverse transcriptase polymerase chain reaction. Since no mutation could be detected, a multiple-ligation probe assay was performed. Though genetic testing was negative for mutations in the NF-1 gene, the patient was diagnosed with NF-1 since he fulfilled the diagnostic criteria. ${ }^{3}$ He had developed $>2$ simple or 1 plexiform congenital or developmental neurofibroma, he showed $>5 / 15 \mathrm{~mm}$ "Café au lait" spots, and he had developed skeletal abnormalities. ${ }^{3}$ He did not have axillary or inguinal freckles, no pigmented harmartomas of the iris (Lish nodules), no optic gliomas, and no affected first-degree relative.

After repeated pleural punctures, carried out by pulmonologists, excess pleural fluid was interpreted as lymph fluid and a chylothorax was diagnosed. Search for causes of the chylothorax revealed a stenosis of the left subclavian vein at the site where the thoracic duct enters the vein. Though recommended, lymphography was not carried out. For the chylothorax, talcum was injected into the pleural sinus, initially with only a transient effect. After repeated drainage of the chylothorax and repeated talcum injections, however, a permanent beneficial therapeutic effect was achieved. Leg edema did not increase after talcum injections into the pleural sinus. Also, ligation of the thoracic duct at the level of the diaphragmatic hiatus was considered but not carried out. It was recommended that he wear compression stockings to control leg edema, but he did not follow these instructions.

\section{Discussion}

NF-1 is a neurocutaneous disorder (RASopathy, phakomatosis), which affects the neuroectoderm as well as the mesoderm. ${ }^{3}$ Frequent clinical manifestations of NF-1 include cutaneous lesions, skeletal abnormalities, and multiple nerve sheath 
tumors. ${ }^{4,5}$ Three types of neurofibromas are found in NF-1 patients: localized neurofibroma, plexiform neurofibroma (involving an entire extremity), and diffuse neurofibroma. ${ }^{6}$ More rarely, patients with NF-1 exhibit vasculopathy affecting the arteries, ${ }^{1}$ the veins, or even the lymph vessels. ${ }^{1,7,8}$ Affliction of the mesenteric artery or vein may coincide with marked thickening of the intima due to spindle cell proliferation, ${ }^{8}$ and may result in protein-losing enteropathy by altering the hemodynamic state and microvascular permeability in the intestines. Impairment of the superior mesenteric artery in NF-1 has also been previously reported by others. ${ }^{9}$ The vascular abnormalities in our patient were interpreted as a manifestation of the underlying genetic defect. In diagnosing NF-1, an individual must meet two or more of the following criteria: six or more CALM, two or more neurofibromas or one plexiform neurofibroma; freckling in the axillary or inguinal regions; optic glioma; two or more Lisch nodules; distinctive osseous lesions; or a first-degree relative with the diagnosis of NF-1. Patients with Legius syndrome may also meet the National Institutes of Health diagnostic criteria for NF-1 based on pigmentary abnormalities like CALM and distinctive freckling pattern alone but may develop additional phenotypic features. Absence of an NF-1 mutation in the presented patient does not exclude NF-1 and may be due to mosaicism which could be confirmed by investigating tissues other than blood cells. An indication for mosaicism could be the half-sided occurrence of the CALM.

Though affliction of the lymphatic vessels in NF-1 is regarded as rare, ${ }^{10}$ it has occasionally been reported. ${ }^{2,7,8,11}$ Lymphangiopathy frequently manifests as leg edema. Only in a single patient has pericardial effusion been described as a consequence of lymphangiopathy. ${ }^{7}$ In a report from Japan, the authors described affliction of the thoracic duct in the form of a cyst, which ruptured and resulted in a chylothorax. ${ }^{2}$ Another paper reported the occurrence of severe diarrhea in a 44-year-old male with NF-1 resulting in progressive hypoproteinemia. ${ }^{8}$ Diarrhea in this patient subsided not earlier than after ileocecal resection. Macroscopic inspection of the resected ileum revealed marked edema of the wall and a pseudomembranous enteritis-like appearance of the luminal surface. Histological examination revealed dilated lymph vessels in the intestinal wall. ${ }^{8}$ Gastrointestinal affliction was also reported in a 26-year-old female in whom capsule endoscopy revealed small-intestinal lymphangioectasia. ${ }^{12}$

Pleural effusion and pericardial effusion in our patient were initially attributed to systemic involvement in erysipelas or bronchitis. After identification of the pleural effusion as lymph fluid, however, the previously suspected pathomechanism was dismissed. Presumably, there was preexisting generalized lymphangiopathy ${ }_{1}^{13}$ which became symptomatic not earlier than after lymph fluid overproduction during the infection. According to this scenario, lymph fluid overproduction in association with lymphangiopathy results in sequestration of the lymph fluid into the pleural cavity, the pericardium, and the legs. A preexisting stenosis of the thoracic duct at the site of the subclavian vein stenosis could have increased the pressure within the thoracic duct with consecutive sequestration of lymph fluid into the cavities. According to another speculation, chylothorax could be explained by a leaky or ruptured thoracic duct. ${ }^{2}$ It is also conceivable that development of the chylothorax simply reflects the progression of the NF-1. There was no indication for malignancy, idiopathic lymphangiectasia with lymph node hypoplasia, or thoracic duct obstruction. Filariasis was unlikely, given the absence of eosinophilia, stool samples negative for worm eggs, and the beneficial outcome after talcum application. Most likely, however, the erysipelas triggered the development of pleural and pericardial effusion since the subject had never previously had such a problem. Leg edema in the described patient could be also due to compression of paravertebral lymph vessels by the paravertebral fibromas. However, all explanations of the leg edema remain speculative. Legius-syndrome (CALM with/without freckling but absence of neurofibromas, optic pathway gliomas, iris Lisch nodules, or bone abnormalities) $)^{14}$ was excluded upon the clinical presentation (presence of neurofibromas, presence of bone abnormalities). There was also no indication for a heart defect, or cognitive impairment, which are frequent phenotypic features of RASMAPK (RAS-mitogen-activated protein kinase) disorders. ${ }^{14}$ Contrary to NF-1, there is no increased risk for malignancy in Legius syndrome. Other features Legius syndrome shares with NF-1, such as macrocephaly, lipomas, learning difficulties, Noonan syndrome-like facial features, attention deficit hyperactivity disorder (ADHD), or pectus excavatum were also absent in our patient. ${ }^{15} \mathrm{NF}-2$ was excluded upon absence of bilateral vestibular schwannomas, spinal cord schwannomas, meningiomas, or ependymomas, and absence of juvenile cataracts. ${ }^{16}$ Possibly, the vascular abnormalities of our patient were not causally related to NF-1 and represent a mere coincidental finding.

This case suggests that NF-1 may affect, in addition to the arteries and the veins, also the lymph vessels resulting in impaired lymph fluid flow and sequestration of lymph fluid into the pleural sinus, possibly the pericardium, and the legs, triggered by an infection. Reduction of lymph 
fluid production by diet or palliative injection of talcum into the pleural cavity may have a beneficial effect on the chylothorax.

\section{Author contributions}

JF designed the study, performed the evaluation of the data, and wrote the first draft of the manuscript. CS, ES, and ST were responsible for the analysis and interpretation of the clinical data. All authors revised and approved the final manuscript.

\section{Acknowledgment}

All authors declare that written informed consent was obtained from the patient for publication of this case report and accompanying images.

\section{Disclosure}

The authors report no conflicts of interest in this work.

\section{References}

1. Oderich GS, Sullivan TM, Bower TC, et al. Vascular abnormalities in patients with neurofibromatosis syndrome type I: clinical spectrum, management, and results. J Vasc Surg. 2007;46(3):475-484.

2. Mizumura S, Ohata M, Abe S, Yamaguchi S, Muranaka S. [Surgical case of thoracic duct cyst with generalized neurofibroma.] Kyobu Geka. 1968;21(1):57-60. Japanese.

3. Sullivan TP, Seeger LL, Doberneck SA, Eckardt JJ. Case report 828 : plexiform neurofibroma of the tibial nerve invading the medial and lateral gastrocnemius muscles and plantaris muscle. Skeletal Radiol. 1994;23(2):149-152.

4. Reynolds RM, Browning GG, Nawroz I, Campbell IW. Von Recklinghausen's neurofibromatosis: neurofibromatosis type 1. Lancet. 2003;361(9368):1552-1554.
5. Stull MA, Moser RP Jr, Kransdorf MJ, Bogumill GP, Nelson MC. Magnetic resonance appearance of peripheral nerve sheath tumors. Skeletal Radiol. 1991;20(1):9-14.

6. Hourani R, Rizk T, Kung S, Boudghène F. Elephantiasis neuromatosa in neurofibromatis type I. MRI findings with review of the literature. J Neuroradiol. 2006;33(1):62-66.

7. Itkin M, Swe NM, Shapiro SE, Shrager JB. Spontaneous chylopericardium: delineation of the underlying anatomic pathology by CT lymphangiography. Ann Thorac Surg. 2009;87(5):1595-1597.

8. Tatemichi M, Nagata H, Morinaga S, Kaneda S. Protein-losing enteropathy caused by mesenteric vascular involvement of neurofibromatosis. Dig Dis Sci. 1993;38(8):1549-1553.

9. Mendonça CT, Weingartner J, de Carvalho CA, Costa DS. Endovascular treatment of contained rupture of a superior mesenteric artery aneurysm resulting from neurofibromatosis type I. J Vasc Surg. 2010;51(2): 461-464.

10. Syrbe S, Eberle K, Strenge S, et al. [Neurofibromatosis type 1 and associated clinical abnormalities in 27 children.] Neurofibromatose Typ 1 und assoziierte Krankheiten bei 27 Kindern und Jugendlichen. Klin Padiatr. 2007;219(6):326-332. German.

11. Zochodne D. Von Recklinghausen's vasculopathy. Am J Med Sci. 1984;287(1):64-65.

12. Calabrese C, Pironi L, Di Febo G. Capsule endoscopy revealing small-intestinal lymphangiectasia and GI stromal tumor polyps in neurofibromatosis type 1. Gastrointest Endosc. 2006;64(1):130-131.

13. Pexsters J, Gruwez J, Lacquet A, van Rossum W. [Angiodysplasia in Von Recklinghausen's disease.] L'angiodysplasie dans la maladie de Von Recklinghausen. Phlebologie. 1971;24(2):143-150. French.

14. Denayer E, Descheemaeker MJ, Stewart DR, et al. Observations on intelligence and behavior in 15 patients with Legius syndrome. Am J Med Genet C Semin Med Genet. 2011;157(2):123-128.

15. Sumner K, Crockett DK, Muram T, Mallempati K, Best H, Mao R. The SPRED1 variants repository for legius syndrome. G3 (Bethesda). 2011;1(6):451-456.

16. Davidson TB, Sanchez-Lara PA, Randolph LM, et al. Microdeletion $\operatorname{del}(22)$ (q12.2) encompassing the facial development-associated gene, MN1 (meningioma 1) in a child with Pierre-Robin sequence (including cleft palate) and neurofibromatosis 2 (NF2): a case report and review of the literature. BMC Med Genet. 2012;13:19.
International Journal of General Medicine

\section{Publish your work in this journal}

The International Journal of General Medicine is an international, peer-reviewed open-access journal that focuses on general and internal medicine, pathogenesis, epidemiology, diagnosis, monitoring and treatment protocols. The journal is characterized by the rapid reporting of reviews, original research and clinical studies across all disease areas.

\section{Dovepress}

A key focus is the elucidation of disease processes and management protocols resulting in improved outcomes for the patient.The manuscript management system is completely online and includes a very quick and fair peer-review system. Visit http://www.dovepress.com/ testimonials.php to read real quotes from published authors. 